\title{
Presentación didáctica comparativa del beneficio ecológico- económico del uso de las energías alternativas en hogares comunitarios e infantiles en Barranquilla, en el marco del contexto de sostenibilidad energética
}

\section{Comparative Didactic Presentation of ecological benefit - economic use of alternative energy in Community and children's Homes in Barranquilla, in the context of sustainable energy framework}

Dirección de Investigaciones y Trasferencias en la Universidad Autónoma del Caribe, Magister en Science of Buffalo State University, pbonaveri@uac.edu.co Pablo Daniel Bonaveri

Docente Investigador Universidad Autónoma del Caribe, Maestrante en Energías Renovables luis.mealla@uac.edu.co

\author{
Luis Enrique Mealla Sánchez
}

Recibido: Oct.4 de 2012

Aceptado: Oct 31 de 2012

\section{RESUMEN}

Este artículo hace una revisión de los aspectos más importantes de la situación actual en materia energética, en lo incursionado por los autores en el ámbito de los hogares comunitarios en Barranquilla, desde el paradigma de la investigación acción. En dichos hogares, la capacitación tuvo un enfoque desde la praxis educativa. Muestra no solo la problemática de la situación actual de las reservas probadas de petróleo, carbón y gas natural, sino primordialmente la necesidad de implementar estrategias didácticas de uso alternativas utilizando energía solar, sino la necesidad de tomar conciencia de que la situación en el planeta Tierra hoy es grave y atañe a la supervivencia misma del hombre. Es evidenciar la conciencia socio-ambiental, en una muestra de 295 hogares comunitarios, para presentarles didácticamente la construcción de hornos solares, su manipulación adecuada y el mantenimiento de los mismos. Llevó a entender que el problema es de todos los integrantes de la comunidad y al desarrollo de una cultura con enfoque sistémico, que permita construir, mediante la capacitación, comunidades abiertas al cambio, a los desarrollos tecnológicos y ecológicamente sostenibles.

Palabras Clave: Capacitación en hogares comunitarios, beneficio ecológico- económico del uso de las energías alternativas, reservas naturales, fuentes renovables.

\begin{abstract}
In this article, it makes a review of the most important aspects of the current situation in the energy field, in where authors in the field of community and children's homes in Barranquilla, from the action research paradigm. In households, the training had an approach from the educational practice. Sample not only the problems of the current situation of proven reserves of oil, coal and gas natural, but primarily the need to implement alternative use teaching strategies using solar energy, but need to take conscience that the situation on planet earth today is serious and relates to the very survival of man. This is demonstrate environmental awareness, in a sample of 295 community homes, to introduce didactically building solar ovens, proper handling and maintenance of the same. It led to understand that the problem is all the members of the community and to the development of a culture of systematic approach, allowing build, through training, open communities to change, technological and environmentally sustainable developments.
\end{abstract}

Key words: Training in community homes, ecological-economic benefit of the use of alternative energy in community homes, renewable sources, solar cookers. 


\section{Introducción}

El uso y dependencia de los combustibles fósiles, a nivel mundial , ha llevado a revisar, por un lado, los impactos ambientales asociados, y por otro, las reservas comprobadas de los mismos. Por ello, el presente trabajo plantea posibles escenarios a tener en cuenta, suponiendo que el petróleo y el gas natural se agotan según comportamientos tipo "pico del petróleo" (Fernández, S., Jusmet, J. 2010), para lo cual es necesario contemplar diversas posturas de incursionar socio-educativo, sobre el tamaño de los recursos finalmente recuperables y alternativas para suplirlos. Y aunque recursos como el carbón, supone reservas abundantes como para mantener la oferta energética total procedente de los combustibles fósiles, también es cierto, que el futuro de las emisiones de $\mathrm{CO}_{2}$ dependerá sobre todo del resultado de las políticas que, de una parte, apuntan a un uso creciente del carbón, ( a medida que se agotan el petróleo y el gas natural) y, por otra, las que lo limitan desde la política ambiental.

El nuevo escenario mundial plantea entonces, la necesidad de comenzar a sustituir el uso de combustibles fósiles por fuentes de energía renovables, las cuales son reconocidas como la opción más viable, para dar solución a los problemas sociales y comunitarios, asociados al incremento de la demanda energética, a la necesidad de salvaguardar el medio ambiente y al requerimiento de lograr una mejor calidad de vida.

\section{Metodología}

Ante la realidad, arriba enunciada, se reafirma la necesidad de introducir nuevas técnicas basadas en el uso de energías renovables, que reemplacen a las fuentes fósiles. Es decir, cambiar del paradigma convencional basado en fósiles, en energía nuclear y en centrales hidráulicas, a un nuevo paradigma, basado en el uso de energías alternativas, que facilite un desarrollo social acorde con lo ecológico; y para las comunidades menos favorecidas, que sea además competitivo en lo económico

Comprometidos con lo anterior, desde el Grupo de Investigación en Energías Alternativas, de la Universidad Autónoma del Caribe, se ha venido trabajando, desde el paradigma de la investigación acción, la utilización de la energía solar, desde su aplicabilidad, en la cocción de alimentos con la implementación de estas sencillas técnicas, en comedores y barrios emergentes de la ciudad de Barranquilla.

Unos de los objetivos planteados, fue difundir la cultura de cocción utilizando la radiación solar, mediante la capacitación a líderes comunitarios, madres cabeza de familia y encargados de comedores escolares en el uso.

Para la finalidad anterior, se realizó un incursionamiento en la ciudad de Barranquilla, específicamente a 295 hogares comunitarios, para presentarles didácticamente la construcción de hornos solares, su manipulación adecuada y el mantenimiento de los mismos.
Es una transferencia de tecnología, con estrategias didácticas, que faciliten la cocción solar a las comunidades de sectores deprimidos económicamente de la ciudad de Barranquilla, mediante jornadas de capacitación y pruebas de hornos y cocinas solares con miembros de esas comunidades. Se posibilitó también, que los participantes cocinasen y degustasen alimentos mediante esta técnica, y se recopiló información, acerca de cómo cocinaban en sus comunidades. Se estableció una somera relación entre el gasto en combustible convencional y el gasto de construcción de una cocina o un horno solar (Bonaveri. Mealla. 2010. 93-98).

Se acotó como objetivo secundario de este proyecto, lo relacionado con la optimización de materiales que se utilizaron en la construcción de hornos solares. Estos materiales se pueden conseguir a muy bajo costo en el medio local, pues si no, no se alcanza el objetivo social del proyecto.

Se realizó además una búsqueda en el Departamento Administrativo Nacional de Estadística (DANE, <http://www.dane.gov.co/> 2006) sobre zonas de Barranquilla que presentaran población de muy bajos recursos y que además funcionen organizaciones tales como clubes de madres, comedores infantiles, comedores escuela o asociaciones comunitarias, en la suposición de que este organismo manejaba este tipo de información.

Ante la inexistencia de datos referentes a la población de estudio, con relación al tema de uso de combustibles en dicha comunidad, se recurrió a otros organismos oficiales que trabajan con población emergente, como el Instituto Colombiano de Bienestar Familiar (ICBF, <http://www.icbf.gov.co/portal/page/portal/ PortalICBF > 2006). Por ello, se contó con información referente a madres sustitutas que tienen a su cargo hogares para niños en estado de desamparo y de personas que administran comedores comunitarios, las cuales forman parte muchas veces de estos hogares sustitutos.

A estos datos se les hizo un minucioso estudio a fin de determinar cuáles eran candidatos a la implementación de las actividades de capacitación a líderes comunitarios, para la actividad de la praxis a realizar con el uso de alternativas energéticas para la cocción de alimentas. La base de datos contenía 4.445 hogares comunitarios y 49 hogares infantiles; por razones de proximidad se eligió solamente los hogares que estuviesen ubicados en la ciudad de Barranquilla descartando las otras localidades que forman parte del Área Metropolitana. La depuración dejó 295 hogares comunitarios y 25 hogares infantiles.

\section{Contexto mundial de reservas y consumo energético}

De las estadísticas consultadas, el consumo energético mundial total para el año 2005 alcanzó los 138.900 TWh), con un 86,5\% provenientes del usos de combustibles fósiles, aunque hay al menos un $10 \%$ de incertidumbre en estos datos, lo cual es equivalente a una potencia media de 15 TW. Se debe destacar que no todas las economías a nivel mundial mundiales tienen datos exactos 
respecto de sus consumos energéticos y además, el contenido energético exacto del barril de petróleo o de la tonelada de carbón puede variar con la calidad (<http://www.eia.doc.gov/pub/ international/iealf/Table18.xls> 2008).

\section{Combustibles fósiles}

La estimación del combustible fósil remanente en el planeta depende de la comprensión detallada de la corteza terrestre, la cual, aún es imperfecta. Mientras que la tecnología de perforación moderna hace posible perforar pozos de hasta $3 \mathrm{~km}$ de agua para verificar la composición exacta de la geología, la mitad del océano es sobrepasa en profundidad a los $3 \mathrm{~km}$, dejando así por fuera un tercio del planeta más allá del alcance del análisis detallado. Los informes del Grupo de Vigilancia Energética muestran que las demandas de petróleo no pueden ser cubiertas y "que el recurso uranio estará agotado en 70 años" (http://www.energywatchgroup. org/fileadmin/global/pdf/ewg_oilreport_10_2007.pdfEarth Watch Report Uranium Report. 2008. >,p.p 39-42)

\section{Reservas de Petróleo}

Las estadísticas muestran que las reservas pueden ser del orden de $57 \mathrm{ZJ}$ para el petróleo en la Tierra (aunque esta estimaciones puedan variar en el orden de $8 \mathrm{ZJ}$,[] de las reservas actualmente probadas y recuperables, hasta la máxima de $110 \mathrm{ZJ}$ ) consistente en las reservas disponibles (no recuperables), y que incluye las estimaciones para fuentes no convencionales, de derivados como las arenas de alquitrán y las pizarras bituminosas. El consenso actual, de 18 estimaciones reconocidas de los perfiles de suministro, apunta a que el pico de la extracción se dará en 2020, con una tasa de unos 93 millones de barriles al día. Actualmente, el consumo de petróleo está equivalente a 31,1 mil millones de barriles, lo que indica que el consumo sería de 85 millones de barriles al día (<http://www.opec.org/library/Annual\%20 Statistical\%20Bulletin/pdf/ASB2005.pdf 〉).

Existe consenso, de que el pico de producción de petróleo podría ser alcanzado en un futuro cercano, llegando a un incremento de los precios del petróleo (Russell, G. Ann, D. 2007, 15-18). Un informe emitido en el año 2005 por un Ministerio francés, sugería que el escenario más desfavorable podría suceder en el año 2013 (Ministerio francés de Economía, Industria y Finanzas <http:// news.bbc.co.uk/1/hi/bussines/4077802.stm.2007>). Existen además teorías, que pronostican que el pico podría darse en tan sólo 2-3 años. Las predicciones realizadas por ASPO (Asociación para el Estudio del Pico del Petróleo y del Gas) lo colocaban para el 2010 (La teoría de Hubbert-1956).

\section{Reservas de Carbón}

La Administración de Información de Energía de los Estados Unidos (EIA) declaró que las reservas mundiales de carbón eran de 930 mil millones de toneladas, al menos contando desde el año 2006, lo cual y al ritmo de extracción que se venía dando durarían unos 132 años. Sin embargo, la tasa de consumo de este mineral se ha incrementado entre 2 - 3\% por año, mostrando una tasa de crecimiento en $2,5 \%$, lo cual lleva a que en realidad, y debido al crecimiento exponencial, la proyección sea de unos 56 años (en 2065) (International Energy Outlook 2007. Chapter 5). Ahora, y de acuerdo con informe de US EIA, con el consumo mundial total de energía de 15,7 teravatios, la reserva de carbón suficiente para proporcionar energía a todo el planeta, sería solo de 37 años, esto ignorando la necesidad de transporte de los combustibles líquidos y suponiendo $0 \%$ de crecimiento de la demanda (US EIA http://www.eia.gov/forecasts/steo/special/ pdf/2013_sp_01.pdf 2011).

Según datos de British Geological Survey, en el año 2006, el principal productor de carbón con 38\% de participación fue China, seguido por Estados Unidos, la Unión Europea y la India. (http:// www.eia.gov/cfapps/ipdbproject/IEDIIndex3,2010)

En la tabla 3 se presenta los totales de las reservas de carbón por continentes, siendo la más baja las de Sur y Centro América.

Tabla 3: Coal Proved Reserves at end 2010.

\begin{tabular}{|l|c|c|}
\hline \multicolumn{1}{|c|}{ Million tonnes } & Share of Total & R/P ratio \\
\hline Total North America & $28,5 \%$ & 231 \\
\hline Total S. \& Cent. America & $1,5 \%$ & 148 \\
\hline Total Europe \& Eurasia & $35,4 \%$ & 257 \\
\hline Total Middle East \& Africa & $3,8 \%$ & 127 \\
\hline Total Asia Pacific & $30,9 \%$ & 57 \\
\hline
\end{tabular}

Fuente: BP Statistical World Energy Review 2011,

\section{Reservas de Gas}

En "CIA World Factbook (2011)", hace un detalle de las reservas probadas de gas natural de los primeros diez países, las cuales ascienden a 180.650.000.000.000 $\mathrm{m}^{3}$. Las reservas probadas de gas natural en el mundo a inicios del año 2008 eran de más de 180 billones de $\mathrm{m} 3$, Las principales reservas están localizadas en Oriente Medio (39,9\%) y en Europa Oriental CEI (31,8\%), mientras que Europa Occidental sólo posee el 2,9\% de las reservas mundiales (<https://www.cia.gov/library/publications/ the-world-factbook/> 2009).

Con los datos disponibles, hoy en día las reservas evaluadas de gas natural son suficientes para abastecer al mundo.

\section{Del mundo natural autopoiético al mundo artificial, pero sostenible}

Al hablar de combustibles fósiles, reservas y de energías renovables nos lleva a hablar de naturaleza. El término "naturaleza" hace referencia a la razón de ser de los fenómenos del mundo físico, a la vida en general, por lo tanto no incluye los objetos 
Tabla 4: Reservas comprobadas de gas natural por continentes.

\begin{tabular}{|l|c|c|c|c|c|c|}
\hline \multicolumn{1}{|c|}{ PAís } & $\mathbf{2 0 0 6}$ & $\mathbf{2 0 0 7}$ & $\mathbf{2 0 0 8}$ & $\mathbf{2 0 0 9}$ & $\mathbf{2 0 1 0}$ & $\mathbf{\%}$ \\
\hline América del Norte & 7,4 & 7,6 & 8,4 & 9,2 & 9,5 & 4,88 \\
\hline América Central y Sur & 7,2 & 8 & 7,7 & 7,9 & 7,9 & 4,06 \\
\hline Europa Occidental & 5,8 & 5,6 & 6,2 & 6,1 & 6 & 3,08 \\
\hline Europa Oriental y CEI & 57,8 & 57,9 & 53,9 & 54,2 & 58,9 & 30,25 \\
\hline África & 14,2 & 14,5 & 14,6 & 14,8 & 14,8 & 7,60 \\
\hline Oriente Medio & 72,4 & 73 & 73,9 & 75,2 & 77,1 & 39,60 \\
\hline Asia-Oceanía & 14,4 & 15,1 & 15,5 & 16,4 & 20,5 & 10,53 \\
\hline TOTAL MUNDIAL & 179,6 & 181,8 & 180 & 183,7 & 194,7 & 100 \\
\hline
\end{tabular}

Fuente: <https://www.cia.gov/library/publications/the-world-factbook/>2009.

artificiales o la acción directo o indirecta del hombre, a menos que se haga referencia a ello, por ejemplo con expresiones como "naturaleza humana". La naturaleza se extiende desde el mundo de las partículas subatómicas hasta el astronómico.

Debe recordarse, que la palabra "Naturaleza" proviene del latín "natura", que significa "el curso de las cosas, carácter natural"; es la traducción latina de la palabra griega physis, que en su significado original se relaciona con la forma en la que nacen, crecen y se desarrollan los sistemas vivos, los cuales presentan una red de procesos y operaciones, que pueden crear o destruir elementos del mismo sistema, como respuesta a las perturbaciones de su medio $y$, aunque el sistema cambie estructuralmente, dicha red permanece invariable durante toda su existencia, manteniendo la identidad de este, lo cual lleva al concepto de autopoiesis y, concluyéndose podemos decir que los seres vivos son sistemas autopoiéticos al igual que los sistemas naturales.

A menudo se habla también de "entorno natural", referido a los animales salvajes, rocas, bosques, playas, y en general todo lo externo que no ha sido alterado sustancialmente por el hombre, o que perdura a pesar de la intervención de este.

Este concepto más tradicional de las cosas naturales, implica una distinción entre lo natural y lo artificial, entendido esto último como algo realizado por la mente o conciencia humana, con el fin, inicialmente, de satisfacer sus necesidades y mejorar su calidad de vida.

El mundo artificial encierra el estudio de la vida y de aquellos sistemas, que siendo creados por el hombre, exhiben propiedades similares a los seres vivos, a través de modelos de simulación (Christopher Langton http://www.ecured.cu/index.php?title= \%22InternationalConference_on_the_Synthesis_and_Simulation_off_Living_Systems\%22\&action=edit\&redlink $=1$ 2008) fue el primero en utilizar el término, a fines de los años 80 , durante la Primera Conferencia Internacional de la Síntesis y Simulación de Sistemas Vivientes, conocida como Vida Artificial I
También las galaxias, los ecosistemas, los átomos, los organismos vivos, y las comunidades, pueden entenderse como un conjunto de elementos interrelacionados, con una autoorganización que posibilita la existencia del sistema (Bertalanffy, 1976). De aquí, que se podría hablar de tres características estructurales básicas de cada sistema: los elementos que lo componen, las relaciones entre esos elementos, y los límites o fronteras, del propio sistema que determinan si los elementos pertenecen o no a este (Martínez y Requena, 1986. 58).

Aquellos sistemas que presentan límites bien definidos, que actúan como fronteras impidiendo la interacción con cualquier elemento ajeno a este, se los conoce como sistemas cerrados; mientras que la naturaleza de los sistemas abiertos posibilita la interacción y relación con elementos de otros sistemas. Desde esta perspectiva, las comunidades sociales, se comportan más frecuentemente como sistemas abiertos, dependiendo de su interacción con otras comunidades o entes de diversas índoles.

\section{Lo socio educativo en el uso de energía alternativa}

En lo cotidiano, la cocción de alimentos utilizando energía solar, ha mostrado ser tan eficiente como el uso de gas natural o energía eléctrica (B. Capehart, W. Turner, W. Kennedy. 2008). Solo que se requiere un cambio de paradigma, ya que el aceptado es que todo debe realizarse de prisa; y la energía alternativa, puede plantear, nuevas técnicas, como los hornos o cocinas solares, con los cuales los tiempos de cocción, son mayores que con los combustibles tradicionales.

Pero en contraprestación a lo anterior, la ganancia se presenta estriba en la diferencia de costos en la construcción de los mismos, en comparación a los montos ocasionados por el uso de fuentes convencionales, y en ser una técnica amigable con el medio ambiente.

Hay que tener en cuenta, que para los contextos de cualquier índole social, estos son sistemas y todo sistema a partir de su 
propia estructura organizativa, desarrolla un conjunto de propiedades, que no es posible explicar a partir del análisis aislado de las partes que lo forman y que se denominan emergencias.

Esa misma estructura organizativa posibilita el desarrollo de determinadas propiedades que constituyen los límites o fronteras del sistema. La configuración de las relaciones entre los componentes del sistema determina las propias características esenciales, lo que Maturana y Varela (1997), denominaron patrón de organización. En el caso de los sistemas vivos este patrón soporta la existencia de la autopoiesis como una emergencia, como capacidad del sistema para mantenerse o producirse a sí mismo, a partir de una relación dialógica con el medio.

Por su parte, el hombre, como SER social, ha ido modificando su relación con el medio natural en el que vive; en su afán por mejorar la calidad de vida, en función de su necesidades y evolución; ha ido transformando la realidad de acuerdo con sus necesidades y expectativas, creando así lo que con propiedad podemos llamar "mundo artificial" (Gay, A. Ferreras M. 1997), el cual hace parte substancial del ambiente sociocultural y se comporta muchas veces como una verdadera interfaz entre el hombre y el mundo natural.

Existe, la necesidad, desde las instituciones educativas, de formar en competencias acordes a esta interfaz, a este mundo artificial, para no alterar el ciclo bio-físico natural. Pero hoy día, la educación y la misma cultura, marchan por el paradigma equivocado.

Cada sociedad, ha tenido, tiene y tendrá sus teorías y sus suposiciones. No podemos explicar bajo las leyes fundamentales porque deberíamos tener la capacidad de recordar el pasado y no tener el mismo acceso epistémico al futuro. Es un enigma desde el punto de vista de estas leyes: ¿Por qué deberíamos pensar que por actuar ahora, podemos afectar el futuro pero no el pasado? En Latinoamérica nos guiamos por la ideología y estamos obsesionados con el pasado, mientras los asiáticos están guiados por el pragmatismo y obsesionados con el futuro (Oppenheimer, A. 2010. 78). Se ve entonces, la necesidad de innovar desde la educación, a fin de lograr la tan anhelada transformación social y de

Lograr una vida sostenible, con seres ecológicamente cultos, comunidades humanas también sostenibles, que comprendan la habilidad inherente de la naturaleza de sustentar la vida, para después rediseñar nuestras estructuras físicas y tecnológicas, con instituciones sociales en concordancia con esa comprensión (Bonaveri, P. 2010. 76).

Por lo anterior, la capacitación de las personas, era indispensable, y se planeó la actividad, primero llamando a cada uno de los responsables de cada hogar comunitario de la muestra de hogares, con la finalidad de invitarlos a las jornadas de capacitación. A esto, respondieron positivamente solo 40 personas, a las que se les envió una invitación por escrito a nombre de la Universidad, el Centro de Investigaciones y del responsable del grupo de investigación
La capacitación tuvo dos etapas. La primera, consistió en citar a las personas que respondieron a la invitación, trasladarlas desde la sede principal de la Universidad hasta las instalaciones del Complejo Polideportivo donde se realizó la jornada de capacitación.

El entrenamiento, arriba mencionado, estuvo compuesto de charlas magistrales, talleres y prácticas con cocinas construidas con materiales económicos. Se diseñó material gráfico tales como apuntes y folletos a fin de que pudieran reproducir lo aprendido dentro de su comunidad (Nandwani, 2006).

La segunda etapa, consistió en visitas a las comunidades cuyos líderes fueron invitados a la primera etapa de capacitación. Allí se mostró a la comunidad en general el funcionamiento de las cocinas solares tipo caja, comparadas con cocinas de cartón tipo embudo. Se procedió a cocinar un plato típico y se brindó a los asistentes una degustación del mismo.

Por otro lado, se presentó la evaluación y los resultados térmicos de cocinas tipo cajas, realizadas con el objeto de comparar diversos factores constructivos de las mismas utilizando un protocolo de prueba conocido como RICSA (Red Interamericana de Cocción Solar de Alimentos).

Para ello, se dispuso de cuatro cocinas en donde se combinaron dos materiales de placa y dos materiales que sirvieron como aislante. Se sometieron a radiación solar directa calentando la placa colectora y luego al calentamiento utilizando agua como líquido de prueba.

Se procedió a medir las perdidas térmicas y la eficiencia térmica, los parámetros adicionales como el tiempo en que alcanza la temperatura de cocción efectiva; y el tiempo en que la mantiene sin intervención del operador también fueron medidos.

De aquí, el análisis de la información proporcionó un buen rendimiento comparable entre cocinas con placa de hierro y los dos aislantes probados por sobre las cocinas de placa de aluminio; además, por la relación precio-rendimiento, se recomienda su construcción utilizando materiales que tienen alto rendimiento y bajo precio (Bonaveri. Mealla. 2010. 23-28).

\section{Resultados y Análisis}

La utilización de cajas solares para cocción debe ir de la mano de una adecuada capacitación en la construcción y el uso de las mismas o de poder complementar dos técnicas de cocción para el caso de los comedores comunitarios.

Se debe concientizar a la comunidad del problema ambiental y cómo con esta técnica puede aportar solución significativa a la falta de un servicio, a los aspectos cotidianos económicos y ayudar al medio ambiente. 
La comparación del gasto entre la construcción de una cocina solar tipo caja, que oscila entre los 30 y 40 US\$, respectos de los pagos por servicios de gas (un recibo de gas para la cantidad de metros cúbicos que consumen mensualmente en promedio oscila entre los 20 y 25 US\$ mensuales), lo cual, con una adecuada concientización del uso de esta técnica muestra beneficios económicos y ambientales.

Tabla 6: Comparativo de uso de gas natural y construcción de cocinas solares tipo caja.

\begin{tabular}{|l|l|}
\hline Comparativo uso de gas natural y cocción solar & \multicolumn{1}{|c|}{ UU\$ S } \\
\hline consumo de gas anual & US $\$ 300$ \\
\hline construcción cocinas solares & US \$35 \\
\hline
\end{tabular}

Fuente: Los autores. 2010.

La aceptación por parte del grupo intervenido mediante esta alternativa de cocción, fue evidente en el momento que procedieron a degustar los alimentos, notaron que el sabor no es diferente al tradicional.

A continuación se presentan los resultados tabulados y su gráfica, de los tiempos de cocción de los diferentes tipos de cocinas:

Tabla 7. Comparativo del tipo de cocina caja (según construcción) y el tiempo que mantuvieron la temperatura de cocción.

\begin{tabular}{|l|c|c|}
\hline \multicolumn{1}{|c|}{ Tipo de cocina caja } & $\begin{array}{c}\text { Tiempo- } \\
\text { Temperatura } \\
\text { de Cocción }\end{array}$ & Promedio \\
\hline cocinas de aluminio con cubierta simple & $2,5-3,0$ & 2,75 \\
\hline cocinas de aluminio con cubierta doble & $2,0-2,5$ & 2,25 \\
\hline cocinas de hierro con doble cubierta & 2,0 & 2,0 \\
\hline cocinas de hierro con cubierta simple & $1,0-1,5$ & 1,25 \\
\hline
\end{tabular}

Fuente: Los autores. 2010.

Gráfico 1. Comparativo del tipo de cocina caja acorde al material de construcción y el tiempo que mantuvieron la temperatura de cocción

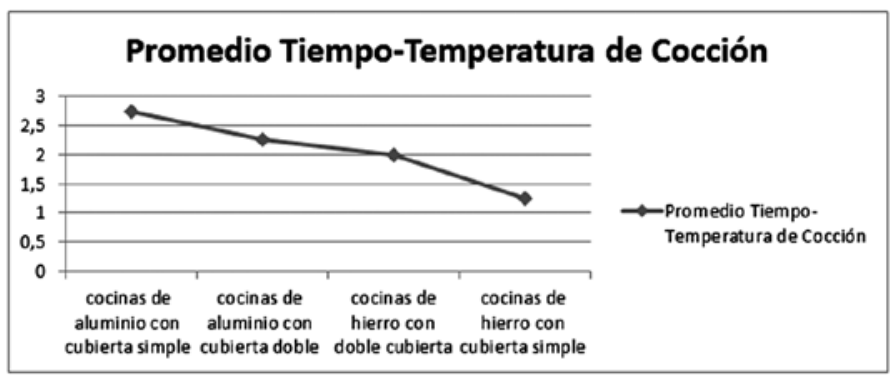

Fuente: Los autores. 2010.

Analizado estos resultados, el tiempo en que las cocinas con carga mantienen la temperatura por encima de la temperatura de cocción, se encontró que las cocinas de aluminio con cubierta simple mantuvieron la temperatura de cocción por el lapso de entre 2,5 a 3 horas. En segundo lugar, las cocinas de aluminio con cubierta doble mantuvieron la temperatura de cocción entre 2 y 2,5 horas. Las cocinas de hierro con doble cubierta mantuvieron alrededor de 2 horas la temperatura de cocción por encima del límite. Al final las cocinas de hierro con cubierta simple solo mantuvieron la temperatura por encima del límite por un lapso entre 1 hora y 1,5 hora.

Estos resultados permitieron mostrarles a las personas de los hogares en la comunidad participante en la capacitación, las ventajas comparativas de un manejo sencillo de un instrumento y técnica para la cocción que estaba redundando a la economía de sus hogares y favoreciendo desde la cotidianidad al medio ambiente

Aspectos Técnicos a tener en cuenta para futuras capacitaciones: De las pruebas realizadas mediante calentamiento simple ( in olla) en las cocinas solares tipo caja, arrojaron resultados interesantes respectos de los valores del coeficiente relativo (Bonaveri. Mealla. 2012).

Para las cocinas de chapa de aluminio pintada de negro con cubierta conformada con un solo vidrio, presentaron más perdidas que ganancias, es decir, que en vacío se enfrían muy fácilmente. El resto de las cocinas, presentaron pérdidas más pequeñas comparadas con la ganancia.

Ahora, analizando las cocinas de chapa de hierro con cubierta simple, presentaron un coeficiente de valor comparable con las cocinas de chapa de aluminio con cubierta doble.

Por otro lado, desde el punto de vista de los precios de las chapas de metal, comparativamente son similares en el mercado.

El rendimiento de las cocinas con chapa de hierro y una sola cubierta presenta un rendimiento térmico parecido. Por su parte, las cocinas de chapa de hierro con doble cubierta presentaron mayor ganancia comparada con las perdidas (lo cual se esperaba en el momento en que se diseñaron las pruebas de campo).

\section{Conclusiones}

Se pudo deducir que, si se extendiese este tipo de estrategias en la cual la utilización de cajas solares para cocción debe ir de la mano de una adecuada capacitación para las personas que conforman hogares comunitarios, en una adecuada construcción y uso de las mismas.

Como se puede complementar dos técnicas similares de cocción para el caso de los comedores comunitarios, a otros sectores que se encontrasen en las mismas condiciones que el grupo con el que se hizo la capacitación, encontraríamos respuestas similares y por sobre todo enriquecedoras. 
El grupo de trabajo, aparte de beneficiarse en estrategias de cocción, aprendió a armar sus propios elementos para cocinar alimentos sin utilizar combustibles tradicionales.

El coeficiente de ajuste entre la potencia estándar y la diferencia de temperatura demostró que si se tiene poca variación de la potencia en función de la diferencia de temperatura entre el líquido de prueba y el ambiente, la perdida de calor hacia el ambiente es menor, por lo tanto las dos cocinas de chapa de aluminio con doble cubierta presentan una perdida menor. Las que le siguen en orden son las de hierro con cubierta doble. Todas las cocinas de cubierta simple presentaron un coeficiente cercano a la unidad; mientras que la de coeficiente más bajo fue la de hierro con papel compactado y cubierta simple.

Al analizar el valor de la potencia estándar para un valor de diferencia de temperatura igual a $50^{\circ} \mathrm{C}$, se observó que las de mayor valor fueron las cocinas de aluminio con cubierta doble, seguidas por las de aluminio con cubierta simple y un poco más abajo estuvieron las de hierro con cubierta doble y muy por debajo las de hierro con cubierta simple.

El gasto de construcción y materiales de cada una de las cocinas, es de aproximadamente entre 30 US\$ para las cocinas de chapa de hierro o aluminio con papel compactado y una sola cubierta y de 50 US\$ para las mismas cocinas pero con cubierta doble. Así mismo las cocinas de chapa de hierro o aluminio con lana de vidrio aproximadamente cuestan 40 US $\$$ si tiene cubierta simple y 60 US $\$$ si tiene doble vidrio en la cubierta. Ante estos datos, comparando precio y rendimiento térmico en vacío, las cocinas de chapa de hierro con cubierta simple son las que mejor desempeño presentan.

Analizando los resultados de las pruebas con agua concluimos que las cocinas de aluminio con cubierta simple presentaron un mejor rendimiento, por encima de las cocinas de hierro con cubierta doble. Por lo tanto, se recomiendan por precio y rendimiento las cocinas de chapa de aluminio pintadas de negro, aplicándole cubierta simple.

Se recomienda el uso de aislante térmico construido a partir de periódico compactado, pues abarata en gran medida los gastos de construcción de los hornos solares.

Para finalizar, cabe destacar, que el consumo energético mundial aumenta constantemente, debido al crecimiento socioeconómico de las naciones y al aumento de la población a nivel mundial. Las reservas mundiales de combustibles fósiles seguirán utilizándose durante muchos años, no obstante la limitante la impone la protección y cuidado del ambiente ante la amenaza del Calentamiento Global.

Esto, sumado a los proyectos mencionados, lleva a retomar lo siguiente:
... hoy los riesgos mayores son endógenos y consecuencia de su propio accionar, por lo que se debe dar paso a un enfoque sistémico, teniendo presente que la actividad humana, tal cual se desarrolla en la actualidad, al no responder al ciclo biofísico natural, es el factor más importante en el proceso de alteración del medio ambiente, que interesa por la magnitud de las modificaciones que puede provocary por la generación de subproductos o residuos potencialmente peligrosos para toda la biósfera, la cual sufre un proceso de degradación cada vez más importante.

Hoy la situación es grave y atañe a la supervivencia misma del hombre en el planeta Tierra, pero los avances de la ciencia, el desarrollo tecnológico, la conciencia socio-ambiental que hoy, en la era del capital intelectual tenemos, muestran un futuro promisorio, para todos aquellos que estén dispuestos a hacer un uso racional de estas, pero cualquier solución pasa primero por la toma de conciencia del problema por todos los integrantes de la comunidad y del desarrollo de una cultura con enfoque sistémico, que permitan construir comunidades abiertas al cambio, a los desarrollos tecnológicos, pero ecológicamente sostenibles, diseñadas de tal modo que sus tecnologías y sus instituciones sociales no interfieran la capacidad inherente a la naturaleza para mantener la vida (Bonaveri, 2010. 122-123)

El enfoque debe ser el de generar y utilizar la investigación, para los desarrollos de la tecnología y modelos que permitan la reconfiguración de zonas urbanas y rurales, creando comunidades con visión sostenible, mediante procesos educativos que afecten en los ámbitos social, económico y ambiental.

Lo anterior, teniendo claro que sostenibilidad significa desarrollo que satisface las necesidades del presente, sin comprometer la capacidad de futuras generaciones de satisfacer sus propias necesidades y para ello es indispensable una educación que los capacite para tal finalidad.

\section{Referencias}

Bonaveri, P. Mealla, L. (2010). Cocción solar - una alternativa para comunidades emergentes de Barranquilla. Avances en Energías Renovables y Medio Ambiente. Vol. 14. Impreso en la Argentina.. p.p..93-98.

(2012). Alternativas de construcción utilizando materiales de bajo costo para la evaluación térmica de cocinas solares tipo caja .Revista ION, 25(1). p.p. 17-23

BP Statistical World Energy Review. (2011). Recuperado de: http://www.bp.com/assets/bp_internet/globalbp/globalbp_uk_english/reports_and_publications/statistical_energy_review_2011/STAGING/local_assets/p df/statistical_review_of_world_energy_full_report_2011. pdf 
British Geological Survey, recuperado en: http://www.eia.gov/ cfapps/ipdbproject/IEDIIndex3, 2010

Capehart, B. Turner, W. Kennedy, W. Eds. (2008). Guide to Energy Management. 6th Edition. The Fairmont Press, Atlanta, GAEarth Watch Report Uranium Report. Energy Watch Group. EWG-Series (1). p.p. 39-42

Christopher Langton (2008). Recuperado en: <http://www. ecured.cu/index.php?title $=\% 22$ InternationalConference on_the_Synthesis_and_Simulation_off_Living_Systems\% 22\&action=edit\&redlink $=1>2008$ )

DANE. (2006). Recuperado de: http://www.dane.gov.co/

Fernández, S. Roca Jusmet, J. (2010) .Agotamiento de los combustibles fósiles y emisiones de $\mathrm{CO} 2$ : algunos posibles escenarios futuros de emisiones. Universidad de Barcelona.

Gay, A. Ferreras M. (1997). La educación tecnológica. Cap. 5, 6, 8, 9 y 10. Red Federal de Formación Continua. Ministerio de Cultura y Educación de la Nación. Argentina.

Ministerio francés Economía, Industria y Finanzas. Recuperado en: <http://news.bbc.co.uk/1/hi/bussines/4077802.stm. 2007

Nandwani, S. S. Puri, S. P. (2006) Physica status solidi. Volume 41, Issue 1, p.p. 199-205.

OPEC (2005) Annual Statistical Bulletin. Organization of Petroleum Exporting Countries (2007). Recuperado de: http://
www.opec.org/library/Annual\%20Statistical\%20Bulletin/ pdf/ASB2005.pdf

Oppenheimer, A. (2010) Basta de Historias. La obsesión latinoamericana con el pasado y las 12 claves del futuro. Ed. Nomos impresores.

Russell, G. Ann, D. (2007). Oil Officials see Limit looming on Production. The Wall Street Journal. p.p. 15-18.

RICSA (Red Interamericana de Cocción Solar de Alimentos). (2010) Recuperado de: http://www.solsolidari.org/ es.html?gclid=CMGekPS-_LQCFQ45nAod6EsA3g

The Financial Times Ltda. Energy Watch Group Oil Report. Recuperado en: http://www.energywatchgroup.org/fileadmin/ global/pdf/ewg_oilreport_10_2007.pdf

US EIA Energy Information Administration. Supplement to January Short-Term Energy Outlook. 2013. Recuperado de: http://www.eia.gov/forecasts/steo/special/pdf/2013_sp_01. pdf

US Energy Information Administration. Supplement to January Short-Term Energy Outlook. 2013. Recuperado de: http://www.eia.gov/cfapps/ipdbproject/IEDIndex3. cfm?tid=1\&pid=1\&aid=2).

BP Statistical World Energy Review (2011): 